Recherches en didactique des langues et des cultures

Les cahiers de l'Acedle

$15-3 \mid 2018$

Quelles médiations en didactique des langues et des cultures?

\title{
Médiations linguistique et culturelle dans le cadre formatif universitaire
}

De la traduction à l'appropriation

\section{Léa Courtaud}

\section{OpenEdition \\ Journals}

Édition électronique

URL : http://journals.openedition.org/rdlc/3703

DOI : $10.4000 /$ rdlc.3703

ISSN : $1958-5772$

Éditeur

ACEDLE

\section{Référence électronique}

Léa Courtaud, « Médiations linguistique et culturelle dans le cadre formatif universitaire ", Recherches en didactique des langues et des cultures [En ligne], 15-3 | 2018, mis en ligne le 01 septembre 2018, consulté le 10 décembre 2020. URL : http://journals.openedition.org/rdlc/3703 ; DOI : https://doi.org/ $10.4000 /$ rdlc.3703

Ce document a été généré automatiquement le 10 décembre 2020.

\section{(c) $(1)$}

Recherches en didactique des langues et des cultures is licensed under a Creative Commons AttributionNonCommercial-NoDerivatives 4.0 International License 


\title{
Médiations linguistique et culturelle dans le cadre formatif universitaire
}

\author{
De la traduction à l'appropriation
}

\section{Léa Courtaud}

1 La réflexion développée dans cet article porte sur les relations entre l'appropriation des langues et des connaissances en contexte formatif universitaire et les rapports à la diversité des langues des étudiants et enseignants. Elle s'inscrit dans une recherche doctorale qui se donne en partie pour objectif de construire une réflexion sociolinguistique et didactique sur les enjeux de la diversité des langues et des cultures dans les situations formatives des étudiants. Pour cela, un des points de départ de mon travail consiste à analyser de quelle(s) façon(s) est prise en compte ou non la diversité linguistique et culturelle dans le contexte universitaire français. Ce contexte est marqué par un long processus d'internationalisation ayant pour corollaire une diversification croissante des publics, d'où l'existence d'un enjeu fort lié au traitement de la diversité linguistique et culturelle. Une des finalités de ce travail serait de pouvoir proposer des pistes de réflexion et de propositions formatives à destination des étudiant.e.s et des enseignant.e.s des universités françaises ainsi qu'aux instances de ces universités en charge des politiques formatives.

2 Les éléments d'interprétation proposés ici ont pu être formulés à partir de réflexions suscitées à la suite d'entretiens réalisés avec des étudiants et des enseignants de différentes UFR de l'Université de Tours ${ }^{1}$ et des étudiants de Français Langue Etrangère ${ }^{2}$ (FLE) se préparant à des études en France. Lors de ces entretiens, les écrits des étudiants tiennent une place centrale, compte tenu de l'importance du rôle de l'écriture dans la formation universitaire : je leur propose que nous commentions ensemble leurs notes, brouillons, devoirs, diapositives, en somme tous types de documents ayant trait à leurs cursus de formation. Très rapidement, du fait aussi de mon propre questionnement, apparait le leitmotiv de la construction de la pensée en différentes langues, des passages de langues à d'autres, de la fabrication du sens, avec, entre, et autour des langues. Si le plurilinguisme oral est plus rare ou tabou (excepté dans les filières de $\operatorname{LLCE}^{3}$ ), les pratiques plurilingues sont elles, bien ancrées/encrées sur le papier. La thématisation des 
activités de transpositions, de traductions, considérées comme étant des formes de médiations, ce que j'expliciterai dans cet article, représente un ancrage permettant de questionner en quoi la diversité des langues est constitutive et inhérente au travail intellectuel ${ }^{4}$ que les étudiants doivent mettre en œuvre dans le cadre de leurs formations.

Les formes de médiation existantes à travers les pratiques linguistiques des étudiants dans des situations d'appropriation formative sont interrogées pour cette recherche, non pas à travers l'institution d'un tiers "médiateur" (Lévy, 2003), mais plutôt à partir d'éventuels processus de médiation entre des univers langagiers, en d'autres termes de mise en relation entre ces différents univers. En explorant plus particulièrement des questionnements sur la traduction à la lumière des observables constitués, il va s'agir de réfléchir en quoi les processus de traduction peuvent relever d'une forme de médiation. Cette médiation, dont la force motrice n'est autre qu'un mouvement de pensée serait ainsi comprise comme un des aspects de tout processus d'appropriation. Ces éléments feront l'objet d'un développement et seront étayés par l'apport de références dans les domaines de la philosophie du langage et de la traduction ainsi que de la didactique des langues et plus particulièrement de l'appropriation en langues.

\section{Méthodologie de recherche et constitution des observables}

4 C'est à travers une méthodologie exploratoire et dans une démarche qualitative et interprétante que j'ai choisi d'élaborer cette recherche. Je cherche à comprendre quelles peuvent être les différentes perceptions et prises en compte de la diversité linguistique et culturelle de la part d'étudiants et d'enseignants, tous acteurs impliqués dans des formations universitaires. Les matériaux sur lesquels s'appuie la recherche sont d'ordre divers et ont été constitués entre 2013 et 2017, pour mon mémoire de recherche en M2 tout d'abord, puis pour ma thèse en cours. J'ai réalisé une quinzaine d'entretiens exploratoires et semi-directifs avec des enseignants de différentes UFR de l'université François-Rabelais et une vingtaine d'entretiens avec des étudiants de la même université ainsi que des étudiants de l'école de langue française dans laquelle j'enseigne. Lors d'une partie des entretiens, j'ai demandé aux étudiants d'apporter quelques unes de leurs productions écrites réalisées dans le cadre formatif: notes, devoirs, brouillons, etc. En outre, j'ai réalisé un questionnaire à l'attention d'enseignants de plusieurs universités françaises portant sur l'accueil des différents publics au sein de leurs formations. Plus largement, la réflexion portant sur la place et le rôles des universités dans les processus d'internationalisation prend appui sur un certain nombre de documents tels que des rapports de recherche (notamment Cohen, 2001 ; Vuilletet, 2005 ; Rhida et Paivandi, 2007 ; Coulon et Paivandi, 2003, 2008), les différentes lois régissant l'enseignement supérieur et la recherche (ESR) et leurs évolutions. Ce travail s'appuie en outre sur différents écrits de recherche, en histoire (Attal, Garrigues, Kouame, Vittu, dir., 2005), en didactique universitaire et sociolinguistique (Debono, 2010) et en didactique des écrits universitaires (Mangiante \&t Parpette, 2011). C'est à partir de la constitution de ce corpus et des travaux déjà publiés que je formule les interprétations ${ }^{5}$ présentées ici. 


\section{Diversité linguistique et culturelle : quelle(s) prise(s) en compte?}

\section{Expériences d'écritures en langues}

\section{Dimensions culturelles et formats académiques}

5 Au cours de mes recherches j'ai fait le choix de m'éloigner des approches culturalistes en didactique des langues et des cultures pour inscrire davantage mon propos dans des approches situationnelles, ces dernières permettant de prendre en compte la diversité des situations et des personnes rencontrées. Relativiser les possibles déterminismes culturels ainsi que les assignations culturelles pouvant aller de pair avec ne revient cependant pas à nier les dimensions culturelles liées aux langues ou présentes dans les philosophies d'enseignement, par exemple. Nous allons voir dans cette première partie comment s'entrecroisent aspects culturels et attentes des enseignants au plan des écrits académiques en les confrontant aux témoignages des étudiants rencontrés.

6 Joe, étudiant britannique titulaire d'une licence en Physique et d'une licence en Philosophie obtenues dans une université canadienne, a pour projet éventuel au moment de notre rencontre d'étudier dans une université française l'année suivante. Dans l'extrait d'entretien reproduit ci-après, alors que nous commentons des traductions de syllogismes qu'il a recopiées, l'étudiant questionne le rapport entre langue et pensée, et plus précisément l'éventuelle part de déterminisme linguistique et culturel qui en découlerait :

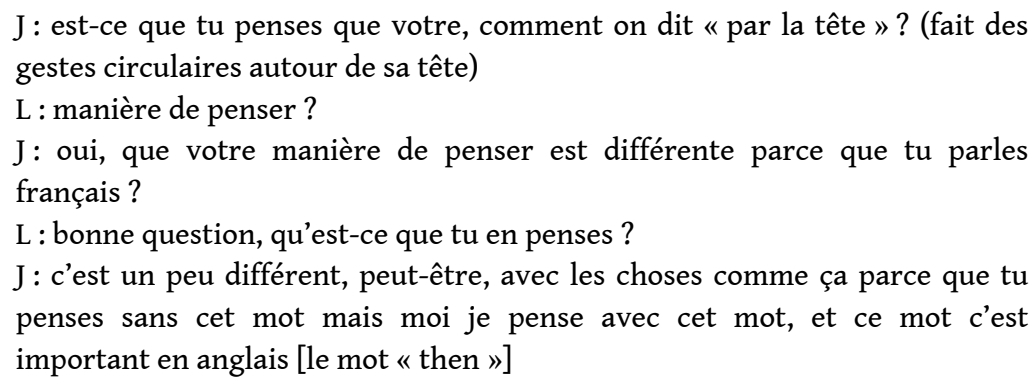

7 C'est un questionnement redondant chez les personnes qui sont sensibilisées aux contacts entre différentes langues et cultures et qui s'interrogent sur les rapports entre langue, culture et manière de penser. Ici, Joe a le sentiment que, sur un même sujet, il pense différemment en anglais et en français. Langue et pensée sont, selon Humboldt (1974), nécessairement liées, non pas dans une vision déterministe de rapports d'une langue reliée à une culture déterminant une façon de penser, mais plutôt à travers l'idée selon laquelle «la langue est l'organe formateur de la pensée ». La formation de la pensée appelée Bildung par Humboldt, fait écho à une forme de culture, culture de l'individu par l'action de sa propre formation, mais aussi culture collective, historique dont il hérite. Associer ces questions d'expériences en langues aux expériences d'écriture des étudiants permet ainsi de mieux envisager le rôle des enseignants dans l'accompagnement à l'écriture des étudiants dans les formations universitaires.

8 En outre il me semble important de souligner les aspects épistémologico-formatifs ${ }^{6}$ de ce que représente écrire à l'université, en particulier dans les cadres d'évaluation. Il y a un 
certain nombre de paramètres historiques et politiques dont il n'est sans doute pas pertinent de faire l'économie. En faisant cela j'essaie d'opérer un changement de point de vue, déplaçant un regard initialement porté sur les plurilinguismes vers la question des relations langue-culture. En effet, se placer uniquement du côté du plurilinguisme sans tenir compte des enjeux contextuels des usages des langues risquerait à mon sens de conforter des visions neutralisantes et utilitaires de la langue. Or je considère que les langues ne sont pas des outils, des vecteurs neutres, interchangeables, et que ce n'est pas chose égale que de s'approprier en français qu'en khmer ou entre ces langues. Réfléchir aux liens entre langue et culture permet de mettre au jour des spécificités en fonction de la langue de formation concernée, dans le cas de nos témoins, le français et l'anglais. Je renverrai à ce sujet aux travaux de J.-L. Chiss (2011) qui éclairent combien la langue française est associée à un contexte historico-culturel en évoquant l'idée de culture $d u$ langage française. Il y met en évidence la référence de centralité de l'histoire culturelle de France pour la langue française et les rapports entre langue et culture apparaissent dans l'intrication entre langue - culture - nation (schéma récurrent dans les discours ministériels sur les universités, voir notamment la dernière citation de cet article). Or certains formats d'écriture (la dissertation par exemple) et manières d'écrire découlent de constructions académiques en prise directe avec l'histoire culturelle française. Il est à noter toutefois que les attentes vis-à-vis des étudiant.e.s varient selon le rapport entretenu par les enseignant.e.s à un certain nombre de normes et de règles.

\section{Ecrire en français, ici et ailleurs : c'est pareil ?}

On s'aperçoit, surtout dans les filières de sciences humaines et de lettres, qu'il y a des représentations fortes et ancrées, sur des façons d'écrire en français «à la française », et j'entends lors des entretiens des étudiants me disant qu'ils apprennent par cœur des phrases, qu'ils imitent des formes écrites (souvent, l'étudiant.e indique comprendre le contenu du cours, tout en ayant conscience que sa façon de formuler dans les évaluations n'est pas acceptée). Gloria, une étudiante seychelloise en M1 de Sciences du langage expose la situation suivante :

G : ici quand je suis arrivée en première année je comprenais pas, je faisais comme j'avais l'habitude de faire dans mon pays, d'après le british way, english way, mais ça passait pas, moi j'avais des mauvaises notes et pourtant j'étais sûre que ce que j'avais écrit c'était des choses, des idées pertinentes, et j'arrivais vraiment pas à comprendre, et quand j'ai demandé à mes profs de première année « mais donnez moi des exemples des écrits, des travaux qui ont déjà été faits par les étudiants ", ben ils m’ont dit «non, on peut pas vous donner ça, mais il suffit de bien structurer vos paragraphes, voilà et...

[...]

$\mathrm{L}:$ mais comment vous avez fait en licence finalement pour...

$G$ : ben, c'était des examens où j'ai appris par cœur alors que c'est vraiment pas ma façon d'apprendre, moi je préfère comprendre et écrire dans mes propres mots mais comme je voyais qu'écrire dans mes propres mots, d'une certaine façon, avec encore l'influence de ma langue maternelle qui cause beaucoup de problèmes, qui a posé beaucoup de problèmes en première année, structure des phrases et tout ça, et que apparemment ce que j'avais écrit c'était pas bien structuré, c'était pas...

$\mathrm{L}:$ mais ils vous ont dit les enseignants pourquoi c'était pas bien structuré ?

$\mathrm{G}$ : ils m'ont fait des commentaires, il y a une prof qui m'a demandé si j'étais anglaise, parce que elle voyait que, elle m'avait dit que en fait les Anglais quand ils écrivent des choses, c'est, on a l'impression que c'est pas bien 
structuré, ils font un peu n'importe quoi, introduction un peu mélangée, mais alors que en français ça doit être bien structuré. Je sais pas si...

$\mathrm{L}$ : ben oui mais je comprends pas, qu'est-ce que ça veut dire, structuré/pas structuré?

$\mathrm{G}$ : ben selon eux c'était ma façon d'écrire qui, les idées c'était pas clair du tout, et pourtant quand ils m'ont posé des questions sur ce que j'ai voulu écrire, moi j'ai pu expliquer donc euh, quand je passe à l'écrit c'est encore plus difficile [...],

L : mais est-ce qu'il n'y a pas un enseignant qui vous a dit « voilà ce que c'est un écrit structuré »?

$\mathrm{G}$ : non, en fait je n'ai pas eu de copie d'examen. Ce que j'ai fait c'est que j'ai demandé à mes copines qui avaient l'habitude d'avoir des super bonnes notes, 16/20, 18/20 parfois, et j'ai lu leur façon d'écrire leurs paragraphes, de bien structurer leurs phrases, et là j'ai compris évidemment, oui, il y avait une différence avec ma façon d'écrire.

$\mathrm{L}$ : d'accord, et comment vous avez pu identifier cette différence, qu'est-ce... $G$ : ben déjà je vois que mes phrases, comme il y avait beaucoup d'interférence avec ma langue maternelle, donc la structure, la syntaxe de la phrase, c'était pas ça en fait que je voulais dire et ça donne une autre idée.

L : d'accord. Alors, parlez-moi des langues que vous mobilisez, vous me parlez de votre langue maternelle,

G : oui, je parle le créole des Seychelles, c'est ma langue maternelle, je parle aussi l'anglais, je le considère comme ma deuxième langue, et après, je parle le français, mais par contre aux Seychelles même si c'est une langue officielle elle n'est pas parlée au quotidien donc euh, pendant que j'étais aux Seychelles j'avais un niveau C1 mais quand je suis venue en France, même avec un niveau $\mathrm{C} 1$ j'avais du mal en première année de communiquer, d'écrire bien en français comme il faut.

10 Dans ce long extrait, l'intrication entre modèles épistémologiques, langues et cultures apparaît clairement. L'étudiante écrit en français pour ses devoirs mais elle conserve dans un premier temps sa manière de faire selon des modèles d'écrits anglo-saxons, cela correspondant aux modèles dans lesquels elle a été scolarisée. Tandis que lui est renvoyé que sa langue maternelle a une influence plutôt négative sur ses productions écrites en français, elle ne reçoit pas de véritable aide pour mieux comprendre les attentes institutionnelles. Elle aura ainsi recours à l'apprentissage par cœur afin de se conformer au mieux aux attentes des enseignant.e.s, et elle demandera de l'aide auprès d'autres étudiantes qui lui prêteront leurs devoirs en guise de modèles. Or selon sa manière d'envisager son projet d'étude, cette étudiante préfèrerait formuler qu'imiter, transformer plutôt que (se) conformer, en d'autres mots, s'approprier (Castellotti, 2017). Si la langue est le lieu-même de l'appropriation, de l'expérience d'appropriation, celle-ci semble néanmoins souvent perçue comme un outil dans de nombreux cas, ce qui explique que beaucoup d'enseignants ne perçoivent pas l'utilité d'expliciter certaines pratiques écrites (formats d'écritures) en les articulant à leur dimension épistémologique.

\section{Usages plurilingues}

\section{Du côté des enseignant.e.s}

11 Dans un premier temps deux tendances ressortent de l'enquête sur la place faite aux langues dans les enseignements, du point de vue des attitudes déclarées des enseignants vis-à-vis des langues autres que la langue d'enseignement (dans notre cas, le français et l'anglais). Certains enseignants disent conseiller aux étudiants de tout «faire» dans la 
langue d'enseignement, c'est-à-dire, prendre des notes, chercher, écrire directement dans cette langue. D'autres témoins indiquent que la pluralité des langues mobilisées par les étudiants est bien présente (avec, par exemple, l'identification d'écritures dans d'autres langues dans les marges des feuilles de notes), avec l'intuition que se joue là quelque chose de significatif dans les apprentissages des étudiants. Mais que faire avec ces langues? Certains des enseignants rencontrés se demandent s'il serait pertinent et comment il serait possible de mobiliser ces éléments afin de les didactiser. En quoi le plurilinguisme des étudiants constituerait-il (ou non) un levier dans leur appropriation tant disciplinaire que linguistique? Ces points de départ nous donnent à réfléchir sur les relations entre les représentations sociolinguistiques des acteurs impliqués et leurs représentations sur ce qu'est former et/ou se former.

Je posai la question suivante dans le questionnaire adressé aux enseignants :

Certains de vos étudiants étudient dans une langue différente de leur langue d'enseignement habituelle. Selon vous : est-ce un atout du point de vue de l'élaboration de leurs connaissances sur les contenus enseignés? Si oui, de quel ordre?

Les réponses sont contrastées. Bon nombre d'enseignants considèrent ainsi que c'est un plus, comme le traduisent par exemple les discours suivants :

Oui, ça aide à la décentration, relativisation, diversité des points de vue, innovation terminologique

Oui, le passage d'une langue à l'autre oblige à se poser des questions de traductions qui interrogent souvent l'implicite des notions d'une langue donnée.

D'autres enseignants estiment en revanche que le plurilinguisme des étudiants ne constitue pas un intérêt voire encore moins un avantage pour les étudiants, comme le montrent les réponses suivantes :

Pas vraiment, à part quand il s'agit d'introduire ponctuellement une dimension comparative.

Je ne pense pas que cela soit un atout en soi.

Ils ont déjà des difficultés à comprendre les concepts dans leur propre langue.

Non, désavantage.

De façon plus nuancée, certains enseignants considèrent qu'il est plus approprié de « tout faire » dans la langue d'enseignement afin d'éviter un appauvrissement des contenus, tout en estimant que pouvoir mobiliser plusieurs langues permettrait aux étudiants de développer des points de vue complexes sur leur sujet ou discipline d'étude.

Je pense aussi à cette enseignante qui, lors de la discussion suite à une communication dans un colloque indiquait qu'elle voyait bien des écritures en « langue étrangère » (LE) ou « langue maternelle » (LM), (tout dépend du point de vue duquel on se place), sur les documents des étudiants mais qu'elle faisait comme si ces éléments n'existaient pas dans la mesure où elle se demandait bien ce qu'étaient ces écrits, ce qu'ils représentaient pour les étudiants et enfin, comment les mobiliser dans un dessein didactique. Il semble que les processus liés au plurilinguisme dans le contexte des études universitaires gagneraient a être explicités auprès des enseignants, certains d'entre eux pointant une difficulté à agir sur ce terrain en raison de leur méconnaissance de ces éléments. 

invisibilisée ? Le contexte socio-historique sous-tendu par des politiques linguistiques est plutôt favorable au rejet et à l'invisibilisation d'autres langues que la « langue-cible ». La France en tant que nation s'est construite autour d'une seule langue, le français, en combattant toutes les autres langues du pays (Lodge: 1997; Baggioni : 1997). Quoi d'étonnant à ce que nos attitudes vis-à-vis des langues adoptent cette tendance à renforcer la langue considérée comme légitime au détriment des autres? Dans le cas des enseignements en sciences et techniques dispensés en anglais, certains des enseignants rencontrés supposent qu'il n'est pas particulièrement pertinent de tenir compte d'autres langues puisque celle-ci est la langue «reine» de ces disciplines. Les représentations sociolinguistiques des enseignants sur les langues d'enseignement sont ainsi à la fois liées à des enjeux d'identification nationale (par exemple, le statut du français en France) et à la construction des champs disciplinaires (par exemple, le statut de l'anglais selon les domaines de recherche), mais aussi à leurs perceptions de ce que sont les langues (langues neutres vs. langues-cultures), ainsi qu'à leurs représentations liées à leurs expériences d'élèves/étudiant.e.s et d'enseignant.e.s : quels parcours d'apprentissage des langues? Quelles expériences en langues?

\section{Du côté des étudiant.e.s}

Lorsque l'on aborde le sujet des pratiques linguistiques avec les étudiants, il ressort que l'ensemble de ces étudiants mobilise des pratiques plurilingues, que ce soit lors de lectures, de recherches documentaires, de prises de notes, ou de préparations de travaux écrits.

Regardons ces quelques extraits de prises de notes d'une étudiante du $\operatorname{CUEFEE}^{7}$ qui suit des cours à l'université dans différents parcours de L3 dans l'UFR de Lettres et langues. Dans ce premier extrait, l'étudiante suit un cours de méthodologie:

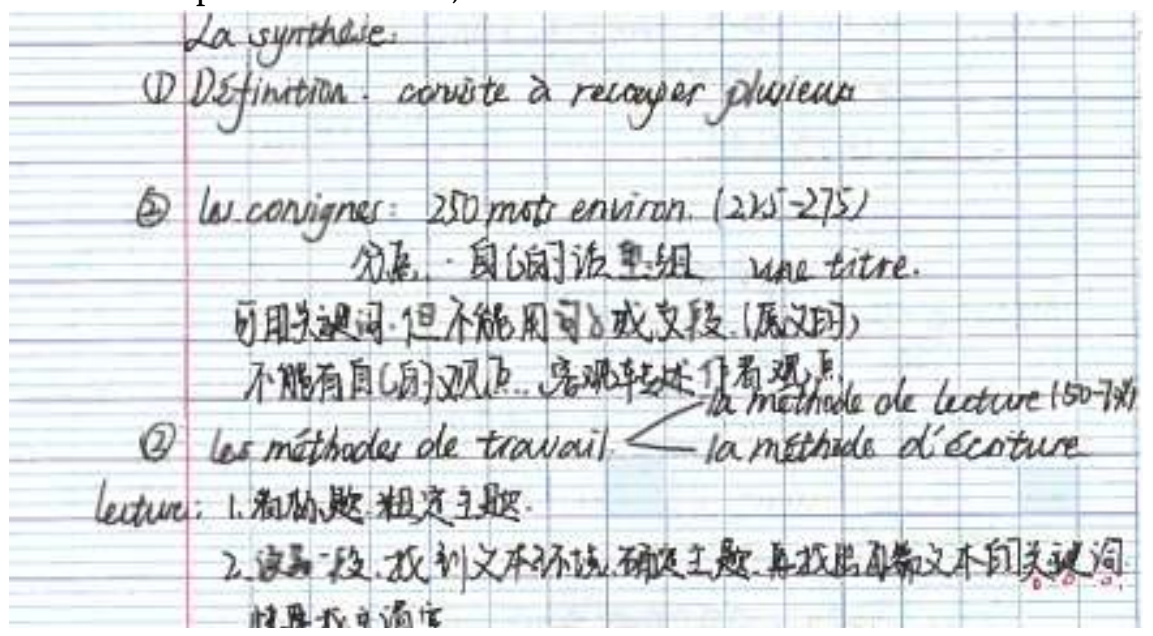

Ici, l'étudiante n'a pas le temps de prendre des notes en français et va donc noter le développement des explications en chinois. Elle traduit simultanément les explications de l'enseignant qu'elle note en chinois.

Dans l'extrait suivant, l'étudiante rencontrée mobilise quatre langues pour prendre des notes lors d'un cours de langue: ikwere (langue enseignée dans le cours), chinois, français, anglais : 


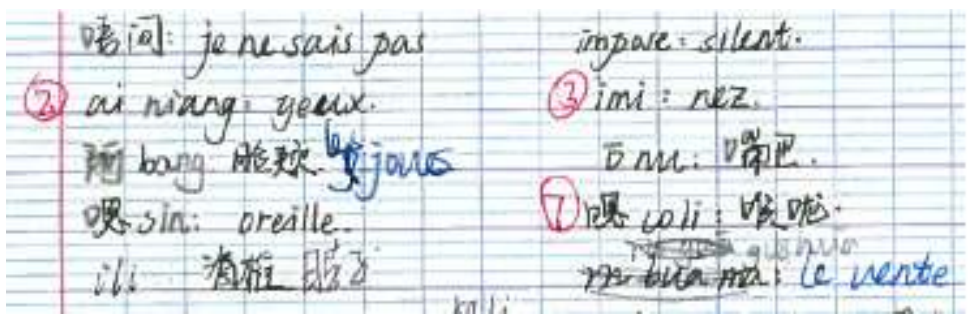

18 Le chinois, le français et l'anglais lui permettent de noter des éléments de sens (définitions) et de prononciation selon un pont réalisé éventuellement par l'étudiante entre chaque langue. Ou encore, comme dans l'extrait de ce cours de traduction de l'anglais vers le français reproduit ci-après, elle note des informations dans la marge :

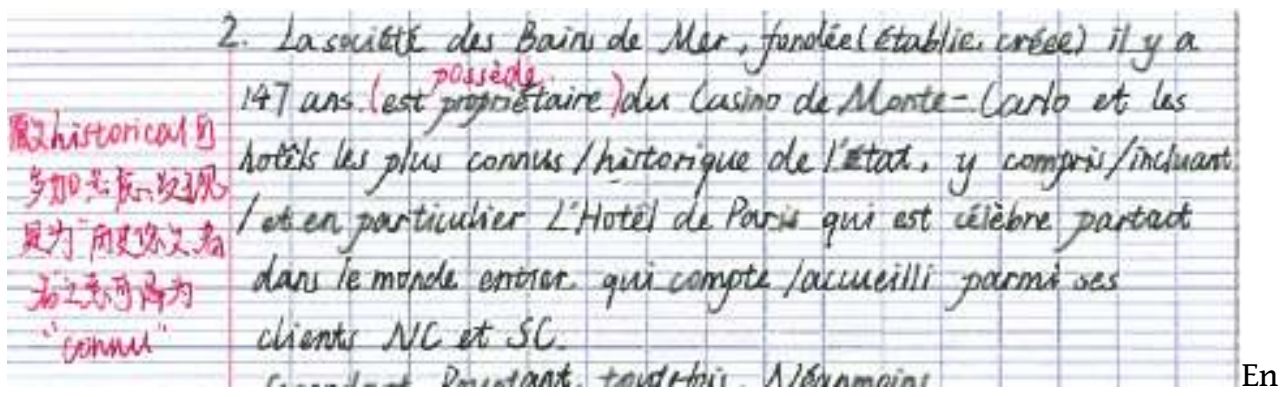

outre les étudiants explicitent sans difficulté leurs pratiques plurilingues quotidiennes. Voici deux extraits d'entretiens au cours desquels Pablo, étudiant en Master de Littérature comparée et Bianca étudiante en Master de Sciences de l'éducation s'expriment au sujet de leurs pratiques plurilingues dans le cadre de leur formation :

$\mathrm{P}$ : donc ben maintenant je me trouve à faire toujours des lectures en français, toujours écouter, comprendre, essayer de m'exprimer en français et parfois je dois revenir un peu dans ma langue pour trouver des mots, pour trouver des concepts et après faire la traduction, faire le point en français, et...

B : y'a encore plein de... d'expressions, de mots, des choses que je connais pas donc quand je lis je comprends presque tout mais y'a toujours des mots à chercher, y'a des nuances que je comprends pas vraiment, donc des mots qui se ressemblent et qui pour moi sont presque pareils mais pour un Français c'est pas du tout la même chose, voilà, ou en tout cas pour les professeurs c'est pas du tout la même chose

Dans la plupart des observables réunis pour cette recherche, j'observe des phénomènes de traductions et de transpositions des idées en différentes langues. Les enseignant.e.s rencontrés sont diversement sensibilisé.e.s aux pratiques plurilingues, que ce soit dans la sphère privée ou professionnelle. Les parcours des témoins ont une influence sur leurs façons de vivre leurs rapports aux langues. Tandis que certains témoins estiment que le plurilinguisme n'est pas un problème, voire est profitable, d'autres paraissent plus désintéressés de ces questions et quelquefois réticents vis-à-vis des pratiques plurilingues. La langue première a-t-elle une influence négative sur les usages des autres langues? Cette question qui a largement été traitée par V. Castellotti (2001a, 2001b), demeure prégnante. Il me semble que le rôle de ces usages pluri/translangagiers gagnerait à être explicité pour donner la possibilité à ceux qui le souhaitent de mieux intégrer ces pratiques plurielles à leurs enseignements et pour pouvoir expliciter leurs fonctions potentielles auprès des étudiants. Nous allons voir comment, à travers les mises en récit de ces pratiques, le traduire prend un rôle médiateur dans les processus 
d'appropriation. On interrogera notamment comment des expériences comme celles du décentrement ou de la mécompréhension permettent l'appropriation dès lors que le sujet fait sens de son vécu.

\section{Le rôle médiateur de la traduction dans le cadre de l'appropriation}

20 Avant de proposer quelques aspects de la traduction comme modalités constitutives de processus d'appropriation pour les étudiant.e.s, il apparaît nécessaire d'exposer les raisons de ce choix: pourquoi user du terme "appropriation" pour évoquer des opérations généralement considérées comme étant de l'ordre de l'apprentissage ? Le cas de la traduction en est un exemple : c'est un exercice dans lequel, comme nous allons le voir ensuite, la personne qui traduit «n'apprend pas » immédiatement (l'apprentissage serait ainsi l'idée d'apprendre de nouveaux savoirs, proposés sous formes d'objectifs, par exemple), mais plutôt, construit du sens à partir de son interprétation du texte: elle va devoir négocier entre son déjà-connu et les nouveaux éléments qu'elle doit intégrer à sa production de sens, et c'est en cela qu'elle se trouve en situation d'appropriation ${ }^{8}$. La notion d'appropriation permet de se concentrer sur l'expérience et le sujet tandis que la notion d'apprentissage se focalise sur des contenus à assimiler (Castellotti : 2015, 2017); or un des choix épistémologiques de ce travail consiste à considérer la dimension expérientielle de la connaissance et des langues comme point de départ pour conceptualiser l'humain qui est au cœur de la recherche en didactique et en sociolinguistique.

\section{Des expériences de décentrement et de mécompréhension}

21 Le fait de commenter avec les étudiants leurs différentes pratiques de traduction permet de rendre compte de l'expérience d'appropriation qui en résulte partiellement. L'exemple le plus frappant est celui de Joe, l'étudiant de FLE qui étudie la philosophie en français. Il me montre quelques uns de ses "French writings", et nous comparons ensemble des traductions de syllogismes :

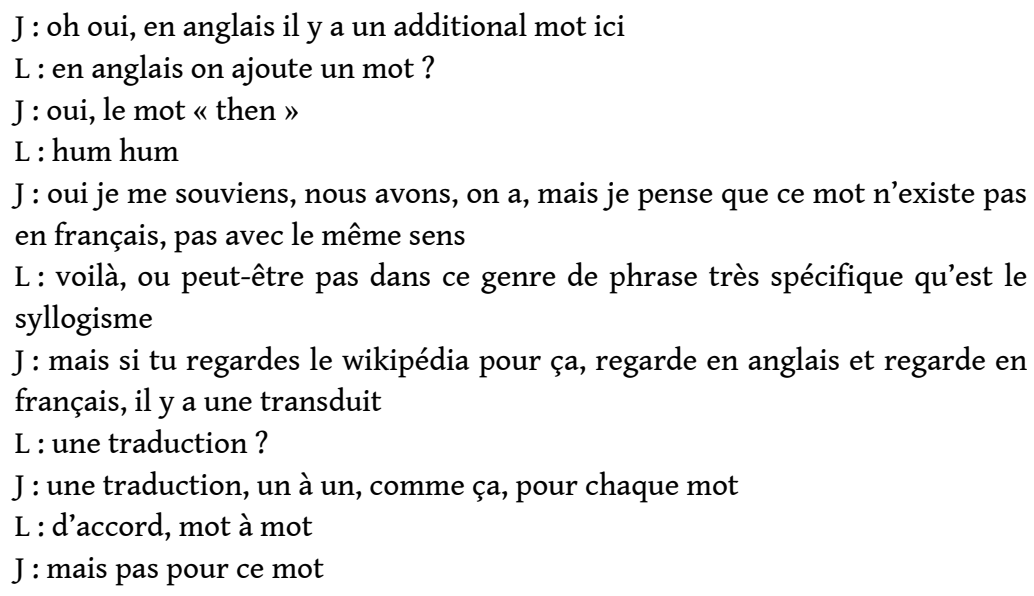

Pour le témoin (dans la citation supra), l'absence de traduction du connecteur « then » est significative parce que «c'est un mot important en anglais ». La citation de M. de Carlo 
qui suit me semble bien illustrer l'expérience du décentrement que vit Joe à partir du travail sur des traductions. Elle y fait référence à Gadamer :

la traduction ne constituerait plus, comme l'a souligné Gadamer, une réponse à un échec, mais un lieu privilégié de rencontre, du moment " qu'elle n'introduit pas seulement une certaine conscience de la diversité des langues, mais également celle de l'altérité des mondes» (Renken 2002 : 7). L'irréductibilité des «intraduisibles » d'une langue constituerait donc la condition même pour expérimenter l'altérité et le «décentrement ", en passant par la dimension du langage (M. de Carlo, 2006 : 124-125)

Pour Joe, étudier la philosophie en mobilisant des traductions est une stratégie d'apprentissage et d'appropriation, pour mieux comprendre le fonctionnement des syllogismes ou encore une notion de philosophie comme nous allons le voir par la suite. Outre les outils linguistiques qu'il doit continuer de s'approprier pour penser et s'exprimer en français, il prend conscience de la dimension transformatrice, dans l'élaboration des connaissances et la façon de les envisager, que peut apporter la comparaison de traductions en différentes langues (ici, l'anglais et le français). La réflexion suscitée, pour l'étudiant comme pour moi, pose l'idée de la traduction en tant qu'élément médiateur du sujet au monde, en particulier du fait de la mise en langage de l'expérience (cf. constats de Joe sur le sens et les mots de la traduction), opération nécessairement réflexive. Ces constats de l'étudiant font écho à la philosophie de cette recherche qui s'appuie notamment sur une vision expérientielle et diversitaire du monde et des langues, à travers laquelle V. Castellotti $(2017: 34,36)$ propose de penser la notion d'appropriation.

Son expérience de l'écriture en français permet également à l'étudiant de se rendre compte de ce que L. Gajo $(2003,2006)$ nomme densité du contenu disciplinaire, et opacité de la langue L'étudiant formule qu'il pensait avoir compris la notion de contrefactualité en anglais, grâce au fait qu'il se sente familier de la langue d'apprentissage, sa langue maternelle et de scolarisation. Cependant, quand il s'essaie à écrire en français au sujet de cette notion, il fait le constat que sa compréhension était peut-être erronée, il se sent finalement en situation de mécompréhension face à la notion. Je lui demande ce qui lui permet d'affirmer cela :

$\mathrm{J}$ : hum, c'est pas... quand j'écrivais ça, j'ai réalisé que je ne comprends pas la contrafactualité comme je, oui, comme je pensais

$[\ldots]$ $\mathrm{J}:$ comment on dit l'oiseau qui répète?

$\mathrm{L}:$ le perroquet

$\mathrm{J}$ : oui, je pense que je suis juste un perroquet avec cette idée. Mon professeur m'a dit que quelque chose..., et j'ai parroté juste comme ça

$\mathrm{L}:$ tu as répété

$\mathrm{J}$ : oui oui oui oui, et j'ai pensé que je comprenais mais quand je ne peux pas juste faire de parroter parce que la langue change, je réalise que non.

Plutôt que de densité et d'opacité du discours, l'idée de mécompréhension telle que la développe Schleiermacher fait davantage référence à un processus de pensée et correspond mieux à l'approche déployée dans le cadre de cette recherche, car elle est propice à la subjectivation des situations observées. À propos de cette mécompréhension dont Jo fait l'expérience, $\mathrm{C}$. Berner écrit dans la présentation de l'ouvrage regroupant différents textes de Schleiermacher : 
[p]our Schleiermacher on ne traduit que des discours, c'est-à-dire de la pensée : celui qui parle ou écrit doit apporter quelque chose à la langue, ce qui interdit la simple répétition. Cela exclut la pensée où n'intervient pas la force de juger : je dois penser par moi-même, ce qui signifie que je ne saurais me contenter de répéter une langue donnée. (Schleiermacher, 1985, [1999] : 15)

La pensée de l'auteur appuie l'idée selon laquelle la traduction, qui est une forme de médiation en ce qu'elle impose l'élaboration d'une pensée, permet au sujet d'exprimer une interprétation, élément constitutif de toute forme d'appropriation, si l'on considère que s'approprier revient à construire du sens en ayant pu intégrer de nouveaux éléments à son propre réseau de significations (Castellotti: 2015, 2017). Dans cette optique appropriative, les personnes produisent un sens toujours nouveau, relatif à leur historicité et à leur être-au-monde.

Il attribue à la "non-compréhension » un rôle déterminant dans le rapport au savoir que va développer le sujet « sachant qu'il sait " ${ }^{9}$ - et qui se rend compte qu'il mécomprend ${ }^{10}$-, tout en reliant la situation de mécompréhension au fait même de la diversité linguistique et culturelle :

[1]a non-compréhension - ou la mécompréhension - est à l'origine de tout processus de compréhension et d'interprétation. En effet, nous ne savons jamais si l'identité des mots dans la bouche de locuteurs différents signifie une conformité d'idées (Schleiermacher, 1985, [1999]:17)

Les phénomènes de mécompréhension font donc référence à un processus de pensée et ne sont pas réductibles à des situations d'échecs communicationnels ou dits «culturels » : un peu différemment de la réhabilitation du préjugé par Gadamer (Debono: 2012), Schleiermacher (ré)habilite l'état de fait de la mécompréhension comme étant l'originemême de la recherche et du développement de la pensée.

\section{Exercices de la traduction, ou de l'interprétation comme modalité d'appropriation}

Non réduite à une technique, la traduction nécessite une interprétation, l'interpréter étant au centre du problème herméneutique longuement développé par Gadamer dans son ouvrage Vérité et Méthode, les grandes lignes d'une herméneutique philosophique (1976). Selon l'auteur, le problème herméneutique est donc celui que pose, non pas la véritable maitrise d'une langue, mais la qualité de l'entente sur quelque chose, à laquelle on parvient dans ce milieu qu'est la langue. Cette première idée exclut la technicité des langues comme dimension essentielle à maitriser, idée à laquelle il me semble que je peux opposer nombre de discours d'enseignants sur la maitrise et la possession de la langue comme faisant partie des clés d'une réussite académique. Ainsi, la maitrise de l'orthographe par exemple apparaît comme étant du domaine des règles et non pas du domaine de la pensée, qui à mon sens, est le lieu de l'appropriation. En outre, pour Gadamer (v. également pour Schleiermacher), le traduire est l'un des modèles de l'interprétation et le sujet, à travers l'activité de traduire, pense :

Interpréter et comprendre, cela signifie le dire avec mes propres paroles. C'est pourquoi la traduction est le modèle de l'interprétation, parce que traduire nous contraint non pas seulement à trouver un mot mais à reconstruire le sens authentique du texte dans un horizon linguistique tout à fait nouveau; une traduction véritable implique toujours une compréhension qu'on peut expliquer. (1982 : 45), L'Art de comprendre. Herméneutique et tradition philosophique, tome 1, cité par M.-A. Vallée, (2011: 133).

Recherches en didactique des langues et des cultures, 15-3 | 2018 
Comme explicité plus haut, la traduction et l'interprétation que celle-ci implique place le sujet au centre du processus de la construction de sens. Mais ne peut-on s'approprier à travers ces modalités que si l'on est plurilingue? Par ailleurs, ne peut-on s'approprier qu'à travers les langues? Cette dernière question ne sera pas traitée ici mais permet d'entrevoir la charge importante donnée au langage, que l'on retrouve en particulier lorsqu'on cherche à évaluer cette appropriation alors qu'elle ne peut être contrôlée dans la mesure où elle ne relève pas d'une situation pré-calibrée et reproductible (Castellotti, 2017 : 48). En effet, l'appropriation peut aussi avoir lieu au travers d'autres médiations que langagières (artistiques par exemple) qui ne convoquent pas nécessairement l'expression de l'expérience par le langage.

\section{Traduire, une expérience nécessairement plurilingue?}

31 Les processus de traduction sont tout aussi présents à l'intérieur d'une même langue qu'entre différentes langues: ce sont dans les deux cas des processus relatifs à une interprétation qui résulte de la mise en mots (ou en musique, peinture dans d'autres cas) d'une production de sens. Si la médiation ne concerne pas uniquement les pratiques dites plurilingues, leur étude met en relief des processus d'interprétation chez les personnes dites ou se considérant monolingues, pour reprendre ce qu'écrit Laurent Gajo (2003). On peut, écrit Ricœur,

prendre le terme traduction au sens strict de transfert d'un message verbal d'une langue dans une autre, soit le prendre au sens large, comme synonyme de l'interprétation de tout ensemble signifiant à l'intérieur de la même communauté linguistique. (Ricœur, 1999 : 8)

L'expérience de médiation concerne donc tous les étudiants à partir du moment où l'on considère que tous mettent en œuvre des processus de traduction, au sens large, en tant qu'élaboration et formulation des connaissances. Les expériences de mécompréhension dans les situations formatives sont l'origine des mouvements d'appropriation des étudiants qui se mettent en posture de comprendre et d'interpréter ce qui, initialement, demeurait opaque et incompris. Les traductions, selon D. Thouard (2007), sont des processus de formation de la pensée, processus au cœur du mouvement et du culturel, non pas en tant qu'objet déterminé mais en tant que vision expérientielle en cours d'élaboration.

[L]es traductions, qu'elles soient intra- ou interlinguistiques, nous font excéder notre propre horizon culturel. Plus encore, elles déploient la possibilité intime des cultures comme étant des transitions, des lieux de sédimentation mais aussi de passage. (Thouard, $2007: 35$ )

La question de la traduction ne se pose pas, alors, qu'en termes de langues au pluriel : elle interroge plus généralement les processus d'interprétation, de compréhension, d'appropriation. Cela donne à s'interroger sur les dimensions langagières dans l'appropriation des connaissances, d'une part sur l'impact des rapports aux langues sur les processus appropriation, d'autre part sur ce qui se produit dans, par, et avec les langues quand on étudie.

Quelles seraient donc les relations entre langue-s et pensée dans le cadre de l'appropriation à l'université s'il ne s'agit pas uniquement de « linguistique » et s'il ne s'agit pas non plus uniquement de pratiques plurilingues ayant un impact sur la formation des connaissances? Les remarques du témoin rencontré reproduites plus haut 
(1.1.1.) posent la nécessité d'interroger ce que représentent les langues en tant que perceptions, ambiances (voir notion de Stimmung chez Heidegger), où les concepts de langue, nation, pensée, culture, s'interpénètrent. Au-delà des aspects de la traduction et de la conceptualisation en situations dites plurilingues, la philosophie développée par Humboldt sur le langage et les langues permet d'élargir conséquemment notre champ de réflexion puisque, comme mentionné brièvement supra, la langue serait déjà traduction de la pensée en tant que lieu de sa formulation, y compris dans une même langue : « La langue est l'organe qui donne forme au contenu de la pensée ", (Humboldt, 1974: 192). La dimension réflexive des langues implique la mise en route d'une situation d'appropriation dès lors que le sujet réfléchit au discours qu'il élabore.

C'est ainsi que se construit mon regard sur les liens entre langue, culture et écriture, l'écriture ayant une place bien particulière dans le paysage culturel et scolaire français.

\section{Conclusion}

À travers cette focalisation sur les démarches d'appropriations des étudiants nous remarquons que ces derniers mettent à l'œuvre des processus de traductions qui relèvent de pratiques de médiation. Ces processus de médiations réinterrogent la notion du «tiers " comme médiateur et ne se présentent pas seulement dans des situations « apparemment » plus interculturelles ou interlinguistiques. Il n'y a pas d'instrument de médiation (Lévy, 2003), mais de la pensée qui (se) construit, une médiation configurante en tant que processus relationnel de soi à soi. Par l'identification d'opérations herméneutiques (comprendre, interpréter, traduire), par l'existence non accidentelle de processus d'altérisation dans ces situations (de penser, d'écrire, de parler...), on se rend compte que la diversité est nécessairement constitutive de tout phénomène de pensée, de compréhension, d'appropriation ${ }^{11}$. Or il semblerait que l'on cherche à contrôler la diversité, voire à l'invisibiliser lorsqu'il s'agit de légitimer des pratiques, y compris non conscientisées, à travers des discours monolingues ou des pratiques dominantes de l'écrit, comme les évoque J.-L. Chiss (2011), liées «à la culture du langage française ». L'activité de traduire provoque une nécessaire conversation, tout autant intérieure qu'extérieure, participe d'une mise en relation d'idées dans le but de construire/fabriquer du sens : elle participe d'un mouvement d'appropriation. Ce bénéfice du traduire ne semble pourtant pas reconnu tant les discours monolingues, objectivants (vs non-neutralité, nonintercheangeabilité des langues) sont prégnants. En outre, les concepts de langue-culture et de Bildung permettent de penser les langues en dehors des déterminismes linguistiques et culturels. À partir de quelques exemples de travaux écrits et de discussions avec des étudiants, on s'aperçoit que si certaines dimensions sont de l'ordre du culturel en tant qu'aspects socio-historiques, toute une autre part de la formation des individus relève de la Bildung (v. sens de la langue) et s'insère dans des projets plus larges. Un point de tension entre des formes d'identités formatives et la question de l'appropriation dans le contexte universitaire français surgit. Ce sont des identités empreintes de l'universalisme français (la devise de la Sorbonne en donne un aperçu : " Hic et ubique terrarum », « ici et partout sur la terre »), de plurilinguismes cachés, de pratiques scolaires nationisées (avec par exemple, l'idée d'écriture «à la française »), appuyées sur une linguistique strucuronationiste (Robillard 2007, 2008), tout cela dans un mouvement commun aux universités du monde entier, se retrouvant sur le " marché des diplômes ", en compétition dans une 
économie de la connaissance. C'est ainsi que les exhortations à l'excellence des universités dans leur rôle de représentation des nations sont courantes :

Il est crucial que chaque nation, chaque université puisse construire et affirmer sa forme d'excellence académique pour rayonner aux yeux du monde. (V. Pécresse, 2011, Conférence d'ouverture du G20)

De ce point de vue, des attentes institutionnelles peuvent être perçues et constituer des éléments d'évaluation dans les offres d'enseignement et le déroulement des formations des étudiants, à travers par exemple les statistiques d'embauches suite à l'obtention d'un diplôme. Dans ces perspectives, résultats et employabilité suite aux études sont des critères fondés sur ce que Ch. Taylor (1991) nomme "raison instrumentale», appelé «arraisonnement» par Heidegger (1953 [1958]); on ne s'attache pas aux questions d'appropriation, autrement dit à la place du sujet dans ses modes de rapport au savoir et dans ses projets de vie. Par ces différentes situations les expériences des personnes (et donc leurs langues, leurs plurilinguismes, les rôles de la diversité linguistique et culturelle), ont tendance à être occultées, ignorées. Or dans le positionnement de recherche qui est le mien, l'humain n'est pas considéré au prisme d'une raison instrumentale mais en tant que sujet expérienciant le monde et donc les langues (Robillard, 2008). Comment les étudiants négocient-ils, s'approprient-ils leurs expériences de formations en regard du projet universitaire actuel dont la régulation est majoritairement issue d'un projet économique? C'est une des questions qui continue d'animer ce travail sur les relations entre langues, cultures et connaissances dans le contexte de l'internationalisation des universités.

\section{BIBLIOGRAPHIE}

Attal, F., Garrigues,J., Kouame, T., Vittu, J.-P. (dir.). (2005), Les universités en Europe du XIIIe siècle à nos jours : Espaces, modèles et fonctions. Paris : Publications de la Sorbonne.

Baggioni, D. (1997). Langues et nations en Europe. Paris : Bibliothèque scientifique Payot.

Bange, P. (2014). La philosophie du langage de Wilhelm von Humboldt (1767-1835). Coll. Ouverture philosophique. Paris : L'Harmattan.

Barré-De-Miniac, C., Brissaud, M., Rispail, M. (dir.). (2005). La littéracie, conceptions théoriques et pratiques d'enseignement de la lecture-écriture. Paris, Budapest, Torino : L'Harmattan.

Castellotti, V. (2017a). Pour une didactique de l'appropriation. Paris : Didier

Castellotti, V. (2017b). « Médiation ou confrontation ? Ou : quelle place pour le comprendre et le traduire en didactologie-didactique des langues? ", Communication au colloque international « Médiations en didactique des langues et des cultures », ACEDLE. Bordeaux : 21 janvier 2017

Castellotti, V. (2015). « Diversité(s), histoire(s), compréhension... Vers des perspectives relationnelles et alterdidactiques pour l'appropriation des langues ». Recherches en didactique des langues et des cultures, $\mathrm{n}^{\circ} 12-1$. http://www.rdlc.revue.org/.

Castellotti, V. (2001a). La langue maternelle en classe de langue étrangère. Paris : Clé International. 
Castellotti, V. (2001b). « Retour sur la formation des enseignants de langues : quelle place pour le plurilinguisme? ", Ela, Études de linguistique appliquée, n¹23-124. pp. 365-372. http:// www.cairn.info/revue-ela/.

Castellotti, V. (2000). «Alterner des langues pour construire des savoirs bilingues », Le Français dans le monde, recherches et applications, Actualité de l'enseignement bilingue, numéro spécial janvier 2000. Paris : CLE International. pp. 118-124.

Charlot, B. (1997), Du rapport au savoir. Paris : Anthropos.

Chiss, J.-L., Merlin-Kajman, H., Puech, C. (dir.). (2011). Deux siècles d'enseignements de l'écriture : enjeux, activités, travail du texte. Paris : Riveneuve.

Debono, M. (2012). «Herméneutique interculturelle et didactique des langues-cultures : quelques fondements théoriques pour une didactique des représentations ", in Debono, M. \& Goï, C. (dir.). Regards interdisciplinaires sur l'épistémologie du divers. Interculturel, herméneutique et interventions didactiques. Bruxelles : E.M.E.

Debono, M. (2010). Construire une didactique interculturelle $d u$ français juridique : approche sociolinguistique, historique et épistémologique. Université de Tours, Thèse de doctorat sous la direction de D. de Robillard. 619 p.

De Carlo, M. (2006). « Quoi traduire ? Comment traduire ? Pourquoi traduire ?», in Ela. Etudes de linguistique appliquée, 2006/1 (n¹41), pp. 117-128.

Gadamer, H.-G. (1960). Vérité et méthode. Coll. L'ordre philosophique. Paris : Le Seuil.

Gajo, L. (2006), « Types de savoirs dans l'enseignement bilingue: problématicité, opacité, densité », in Education et sociétés plurilingues, n²0. pp. 75-87.

Heidegger, M. (1953, [1958]). « La question de la technique », in Essais et conférences. Paris : Gallimard.

Humboldt, W.,Von. (1974). Introduction à l'oeuvre sur le Kavi, et autres essais, traduction et introduction de P. Caussat. Paris : Le Seuil.

Kelkel, L. (1958). « Monde et langage : réflexions sur la philosophie du langage de Wilhelm Von Humboldt ». Les Etudes Philosophiques, ${ }^{\circ} 4$. pp 477-485.

Lodge, R.A. (1997). Le français. Histoire d'un dialecte devenu langue. Paris : Fayard.

Levy, D. (2003). «Introduction », Le Français dans le monde, recherches et applications, La médiation en didactique des langues et des cultures, numéro spécial janvier 2003. Paris : CLE International. pp. 10-23.

Mangiante, J.-M. \& Parpette, C. ( 2011). Le français sur objectif universitaire. Grenoble : PUG.

Meschonni, H. (1999). Poétique du traduire. Lagrasse : Verdier Poche.

Ricoeur, P. (2004). Sur la traduction. Paris : Bayard.

Ricoeur, P. (1999). « Le paradigme de la traduction », Revue ESPRIT. pp. 8-19.

Robillard, D., (de). (2008a). Perspectives alterlinguistiques, Vol. 1 Démons. Paris : L'Harmattan.

Robillard, D., (de). (2008b). Perspectives alterlinguistiques, Vol. 2 Ornithorynques. Paris : L'Harmattan. Schleiermacher, F. (1985, [1999]). Des différentes méthodes du traduire et autre texte. Paris : Le Seuil.

Taylor, C. (1991), [1994]). Le malaise de la modernité. Paris : Editions du Cerf. 
Thouard, D. (2007). « Points de passages : diversité des langue, traduction et compréhension », Hermès, La Revue, 2007/3, n 49. pp. 29-36.

Vallée, M.-A. (2013). Gadamer et Ricœur. La conception herméneutique du langage. Rennes : PUR.

\section{NOTES}

1. UFR (Unités de Formation et de Recherche) de Lettres et langues, d'Arts et sciences humaines et de Sciences et techniques.

2. Etudiants de l'école de FLE dans laquelle j'enseigne par ailleurs.

3. Langues, littératures et civilisations étrangères.

4. Travail de recherche, de réflexion, de conceptualisation, de formulation des connaissances.

5. La façon dont je conçois la notion d'interprétation ne pourra être développée ici mais constitue cependant un point fort des choix épistémologiques effectués pour ma thèse.

6. Renvoie aux règles, traditions, canons, normes, méthodes d'élaboration, d'organisation et de communication des connaissances et aux modèles épistémologiques qui sous-tendent la façon dont les disciplines sont enseignées dans les divers cadres formatifs de l'université.

7. Centre Universitaire d'Enseignement du Français pour Etudiants Etrangers.

8. Je renverrai à ce sujet aux chapitres 1 et 2 de la première partie de l'ouvrage cité dans ce même paragraphe : Castellotti, 2017 : 33-70.

9. Voir B. Charlot (1997), Du rapport au savoir.

10. Je remercie Joanna Lorilleux pour cette proposition de formulation et plus généralement pour sa relecture de ce texte et ses retours éclairants.

11. Voir Castellotti, 2017 : 52. Le second chapitre de l'ouvrage intitulé, «Appropriation, donc diversité » impulse un mouvement traduisant l'idée selon laquelle si l'on se trouve dans une situation d'appropriation, alors il y a nécessairement diversité.

\section{RÉSUMÉS}

Le présent article rend compte d'un travail de réflexion portant sur les enjeux de la diversité linguistique et culturelle en contexte universitaire. Que se passe t-il dans, par, et avec les langues lorsque l'on étudie à l'université ? Questionnant l'impact des rapports aux langues sur les processus d'appropriation, les pratiques plurilingues d'étudiants de différents domaines sont interrogées à travers des entretiens et un questionnaire réalisés avec des étudiants et des enseignants d'universités en France. En quoi la diversité linguistique mobilisée peut-elle être considérée comme constitutive du travail intellectuel des étudiants? En quoi l'exemple de la traduction permet-il de penser la notion de médiation comme partie prenante des processus d'appropriation? Les démarches d'appropriation formative des étudiants sont ici analysées en étant mises en perspective avec les attentes institutionnelles perçues par les étudiants, mais aussi avec les interprétations d'enseignants et le projet de l'institution universitaire française.

El artículo presenta una reflexión sobre los retos asociados a la diversidad lingüística y cultural en el contexto universitario. ¿Qué ocurre en, por, y con los idiomas cuando se estudia en distintos idiomas? Durante la examinación del impacto de los informes lingüísticos sobre los procesos de 
apropiación, se cuestionan las prácticas plurilingües de estudiantes de distintos campos a través de diferentes entrevistas y de un cuestionario, realizados con estudiantes y personal docente de distintas universidades en Francia. ¿En qué aspectos podemos considerar la diversidad lingüística movilizada como constituyente del trabajo intelectual de los estudiantes? ¿De qué manera el ejemplo de la traducción nos permite concebir la idea de mediación como parte del proceso de apropiación? Los procesos de apropiación formativa de los estudiantes son analizados en este texto mediante su puesta en perspectiva con respecto a las expectativas institucionales que perciben los estudiantes, con las interpretaciones de docentes y con el proyecto de la institución universitaria francesa.

\section{INDEX}

Keywords : university education, linguistic and cultural diversity, plurilingualism, translation, mediation, appropriation

Mots-clés : formation universitaire, diversité linguistique et culturelle, plurilinguisme, traduction, médiation, appropriation

\section{AUTEUR}

\section{LÉA COURTAUD}

\section{EA 4428 DYNADIV}

Léa Courtaud est actuellement doctorante à l'université de Tours. Sa thèse en sociolinguistique et didactique des langes vise à approfondir la question des politiques linguistiques, culturelles et formatives (explicites ou non) relatives aux contextes universitaires dans lesquels sont présents des étudiants ayant des parcours scolaires et universitaires antérieurs diversifiés. Le traitement de l'hétérogénéité et de l'interculturalité dans les parcours estudiantins est étudié à partir d'une analyse des rapports entretenus aux dimensions linguistiques (notamment à l'écrit) et culturelles par les acteurs concernés. 